between regional and national level was the key to have these interventions happen rapidly.

\section{P1-86 EPIDEMIOLOGY OF CHILDHOOD ROAD TRAFFIC INJURY: IN BANGLADESH YIELD OF THE LARGEST COMMUNITY BASED SURVEY}

doi:10.1136/jech.2011.142976c.79

\author{
${ }^{1,2}$ M D K ul Baset, ${ }^{*}$ A K M F Rahman, ${ }^{1}$ A Rahman, ${ }^{1}$ S M S R Mashreky, ${ }^{2} E$ Towner. \\ ${ }^{1}$ Centre for Injury Prevention and Research Bangladesh (CIPRB), Dhaka, Bangladesh; \\ ${ }^{2}$ University of the West of England, Bristol, UK
}

Introduction Road traffic injuries (RTIs) are a leading cause of morbidity, disability and mortality in low income countries. $93 \%$ of child road deaths occur in low and middle income countries. Good data are needed to raise awareness of the scale of the problem and to develop and target injury prevention programmes.

Objective Investigate the magnitude of and risk factors for childhood RTIs in Bangladesh.

Methodology A cross sectional study was conducted to determine the current childhood road traffic injury situation in Bangladesh. Face-to-face interviews were used. Multi stage cluster sampling was used to select the sample.

Result Nationally representative data were collected from 171366 rural and urban households. In the sampled households 351651 children aged $0-17$ years were identified; 178285 were males and 173366 females. The rate of non-fatal RTI among children under 18 years of age was calculated as 186.55 per 100000 child-year. The highest incidence (216.06/100 000 child-year) was found among the 5-9 years age group. Among the total children with non-fatal RTI $75.3 \%$ were male and $24.7 \%$ were female. The incidence of childhood RTI was found to be three times higher in rural children than urban children. Most of the childhood RTIs were pedestrian injuries. The rate of fatal RTI was 5.97 per 100000 per year among all children. Conclusion The study has confirmed that childhood RTI is a major public health problem in Bangladesh particularly in rural areas. An appropriate prevention programme is urgently required to prevent fatal and nonfatal RTIs in rural areas.

\section{P1-87 SURVEILLANCE OF DEATH FOR WOMEN OF CHILDBEARING AGE: EXPERIENCE REPORT}

doi:10.1136/jech.2011.142976c.80

M Bastos, * J Armond, S Prado. Universidade de Santo Amaro, São Paulo, SP, Brazil

Introduction Since the establishment of policies of humanisation of delivery and to obtain more accurate data on causes of maternal deaths, maternal mortality in Brazil declined. Pregnant women have been given priority in health services. As a result, we started to notice a large number of deaths of women of childbearing age, not pregnant, who now have more difficulty getting care in health services.

Objectives To identify the causes of death in women of childbearing age (10 to 49 years), to prevent them in the Public Health Unit.

Methods Time series from 2005 to 2009 that evaluated the death certificates of women of childbearing age in the region studied. After checking the main causes of death was held discussion with staff of the Unit on shares, which were immediately put into practice (task force for preventive gynaecological examinations, diagnosis of sexually transmitted diseases, and chronic degenerative diseases).

Results There were 386 deaths of women of childbearing age in the period studied. As causes, undetermined and without care (19.2\%), heart disease $10.9 \%, 10.7 \%$ violent death, cerebrovascular events $6.7 \%$, lung diseases (8.2\%), AIDS $3.6 \%$, maternal causes accounted for $2.07 \%$ and $1.55 \%$ neoplasms. The rate of death between 30 and 49 years rose from $79 \%$ in 2005 to $81 \%$ in 2009 .

Conclusions The most common causes of death were undetermined causes, cardiovascular events and violent death. It appears that prevention and health promotion carried out in health units could prevent such events.

\section{P1-88 DECREASE OF RAILWAY SUICIDES BETWEEN 1998 AND 2006: EVALUATION OF THE GERMAN RAILWAY SUICIDE PREVENTION PROJECT}

doi:10.1136/Jech.2011.142976c.81

${ }^{1} \mathrm{~J}$ Baumert, ${ }^{*} \mathrm{~K}$ Lukaschek, ${ }^{1} \mathrm{~S}$ Kunrath, ${ }^{1,2} \mathrm{~N}$ Erazo, ${ }^{1,2} \mathrm{~K} \mathrm{H}$ Ladwig. ${ }^{1}$ nstitute of Epidemiology II, Helmholtz Zentrum München, Neuherberg, Germany; ${ }^{2}$ Department of Psychosomatic Medicine and Psychotherapy, Klinikum rechts der Isar, Technische Universität München, Munich, Germany

Introduction Railway suicides are a suicidal behaviour which strongly impact psychological and socioeconomic aspects of the railway company, its employees and possible eye witnesses. The German Railway Suicide Prevention Project was carried out in 2002 aimed to prevent suicidal acts on German Railway net by a variety of measurements. The present study evaluated the impact of this project on the number of suicides during an observation period from 1998 to 2006. Methods The data base of the present study is derived from the Event Database Safety (EDS), which is the national central registry of all person accidents in the context of the national German railway company covering the entire German railway track system excluding municipal subway providers. We compared the railway suicide rate 4 years before and after starting the project by defining an "index group" (1998-2001) and a "control group" (2003-2006) using Poisson regression with estimating the average percentage change (APC).

Results The absolute number of suicidal events on the railway track system decreased from 1006 in 1998 to 724 in 2006. The mean suicide rate in the control years was $13.9 \%$ (95\% CI 6.9 to 20.4 ) lower compared to the index years $(p<0.001)$. Adjusting for the overall suicide rate attenuated the decline of the railway suicide rate (APC $4.8 \%, 95 \%$ CI 1.8 to 7.8 ) but significance remained ( $p=0.002$ ). Conclusion The present study revealed a favourable trend with decreasing railway suicide rates even taking the overall suicide rate into account. The preventive measurements carried out by the project might contribute to this development.

\section{P1-89 MILITARY POPULATIONS, MILITARY DISEASES: THE DEVELOPMENT OF MILITARY EPIDEMIOLOGY}

doi:10.1136/jech.2011.142976c.82

${ }^{1}$ B Bergman.* ${ }^{1}$ Army Medical Directorate, Camberley, UK; ${ }^{2}$ Scottish Government, Edinburgh, UK

Introduction In the mid $19^{\text {th }}$ century, military health protection began to be informed by epidemiology. This paper examines the development of military epidemiology and its impact on military health policy.

Methods Historical material drawn from military health reports and other sources is used to illustrate long-term trends and developments.

Results The science of military epidemiology can be traced back to the Scottish Enlightenment of the 18th century. At first qualitative and descriptive, the early nineteenth century saw the development of a more quantitative and analytical approach which became a powerful tool in influencing military policy to protect and improve the poor health of the Victorian soldier, who faced disease and environmental hazards far from home. Formal annual reports on the health of the Army, which were instituted in 1859 and continue to this day, have provided a unique picture of long-term health trends in a changing population. Over time, both the nature of the disease threat and the means of health protection changed, and examples will be given of trends in disease and their impact on military operational effectiveness. The military population itself also 
changed as recruits became better nourished and healthier. Modern technology has updated the methodology for data collection, although not necessarily beneficially.

Conclusion Although the military population, its health problems and the methods of data collection have changed over time, the fundamental principle of basing military health protection on sound epidemiology remains constant. The lessons of the past provide evidence on which future planning can be based.

\section{P1-90 SOCIAL INEQUALITIES IN HEALTH AMONG ELDERLY IN A BRAZILIAN SOUTHEASTERN CITY}

doi:10.1136/jech.2011.142976c.83

${ }^{1} \mathrm{M}$ B de Azevedo Barros, ${ }^{*}{ }^{1} \mathrm{P}$ M B Francisco, ${ }^{1} \mathrm{M}$ G Lima, ${ }^{2} \mathrm{C}$ L G Cesar. ${ }^{1}$ State University of Campinas Medical School, Campinas, São Paulo, Brazil, ${ }^{2}$ University of São Paulo, Faculty of Public Health, São Paulo, Brazil

Introduction Social inequalities in health is an important problem in Brazil challenging the public Health System. This is also an issue that affects the elderly population.

Objective The aim of this study was to assess the magnitude of social inequalities in health status, health behaviour and use of health services in elderly.

Methods A population-based cross-sectional study was carried out in 2008 e 2009 involving 1518 elderly residents of Campinas, SP, Brazil (ISACAMP 2008/2009). Social inequalities were assessed by educational level. Prevalence and adjusted prevalence ratios were estimated applying Poisson multiple regression, using svy commands of Stata11.

Results Significant social differences were found between the educational strata. Elderly individuals with a higher degree of schooling consume more alcoholic beverages $(R P=1.94)$, are less sedentary $(\mathrm{RP}=0.72)$, have healthier dietary (1.64) and a lower prevalence of hypertension $(R P=0.80)$, diabetes $(R P=0.71)$, dizziness $(\mathrm{RP}=0.67)$, headaches $(\mathrm{RP}=0.52)$, back pain $(\mathrm{RP}=0.77)$, visual impairment $(\mathrm{RP}=0.57)$ and denture use $(\mathrm{RP}=0.68)$. But, there were no differences in the use of health services in the previous 2 weeks, in hospitalisation or in surgeries in the previous year and in medicine intake in the previous 3 days. Among elderly with hypertension or diabetes, there were also no differences in the regular use of health services and medication.

Conclusion The results showed strong social inequality in this elderly population with significant differences in several health indicators, along with equity in the access of some health service components, suggesting positive effects of the Brazilian Public Health System on promoting health equity.

\section{P1-91 PREVALENCE AND TEMPORAL TRENDS IN THE PREVALENCE OF SMALL INTESTINAL ATRESIA IN EUROPE: A MULTILEVEL ANALYSIS}

doi:10.1136/jech.2011.142976c.84

${ }^{1} \mathrm{~K}$ E Best, ${ }^{* 1} \mathrm{P}$ W G Tennant, ${ }^{1,2} \mathrm{~J}$ Rankin, ${ }^{3}$ Eurocat Working Group. ${ }^{1}$ Newcastle University, Newcastle upon Tyne, UK; ${ }^{2}$ Regional Maternity Survey Office, Newcastle upon Tyne, UK; ${ }^{3}$ University of Ulster, Belfast, UK

Introduction Small intestinal atresia (SIA) is a congenital anomaly characterised by the abnormal closure, discontinuity or narrowing of the duodenum, jejunum or ileum. This study used multilevel regression to examine the total prevalence and temporal trends in the prevalence of SIA in Europe.

Methods Cases of SIA delivered during 1990-2006 and notified to 21 European congenital anomaly registers formed this populationbased case series. Total prevalence and changes in prevalence over time were modelled using multilevel Poisson regression. Heterogeneity between registers was evaluated from the intercept's random component. Inter-regional differences in trends were examined by including random slopes.

Results 1154 SIA cases were reported among 5383099 registered births. Of 1092 singleton cases, 222 (20.3\%) were associated with chromosomal and 227 (20.8\%) with structural anomalies. The prevalence per 10000 births for singleton cases of normal karyotype was 1.6 (95\% CI 1.5 to 1.7$)$ for SIA, 0.9 (95\% CI 0.8 to 1.0$)$ for duodenal atresia and 0.8 (95\% CI 0.7 to 0.8 ) for JIA. There was no significant trend in SIA, duodenal atresia or JIA prevalence over time $(\mathrm{RR}=1.0,95 \%$ credible interval (CrI): 1.0 to 1.0 , for each) but SIA and duodenal atresia prevalence varied significantly between participating registers ( $p=0.03$ and $p=0.04$, respectively). There was no increased risk of SIA in mothers aged $<20$ years compared to mothers aged 20 to 29 ( $R R=1.3,95 \%$ CrI: 1.0 to $1.8 ; p=0.08$ ).

Conclusion This study found no evidence of a temporal trend in the prevalence of SIA, duodenal atresia or JIA although SIA and duodenal atresia rates varied between geographic areas.

\section{P1-92 AN EPIDEMIOLOGICAL STUDY OF TUBERCULOSIS PATIENTS WITH RISK PATTERN OF HIV/AIDS AMONG UNDERPRIVILEGED POPULATION IN NORTH INDIA}

doi:10.1136/jech.2011.142976c.85

V Bhatia, ${ }^{*}$ S Puri, M Thakare. Government Medical College, Sector 32, Chandigarh, India

Introduction Tuberculosis is a major cause of morbidity and mortality in developing countries. National AIDS Control Organization, India has reported $\mathrm{TB}$ as the commonest opportunistic infection (62.3\%) among HIV infected persons.

Methodology The study was conducted among 155 patients of tuberculosis at three Microcopy centres attending urban and rural health centres with the objectives of studying epidemiological profile of patients and to assess HIV-AIDS pattern and high risk behaviour. Information on pres-designed format related to socio-demographic clinical profile, categorisation, treatment and awareness about HIVAIDS, mode of transmission and behaviour was gathered in 2009.

Results Maximum number of patients were in the age group of $21-30$ yrs (23.22\%). $41.93 \%$ being illiterate. $42.58 \%$ were having a monthly family income of under Rs. 3000/- (US\$ 67). $47.74 \%$ had migrated from another poor state, $70.32 \%$ married. $11.72 \%$ TB patients were staying with under-six children. Fever $(79.35 \%)$ and cough $(72.25 \%)$ were presenting symptoms at starting the treatment. $54.19 \%$ belonged to category I of DOTS. Only half (54.19\%) were aware of HIV-AIDS- $77.35 \%$ in rural and $42.15 \%$ in urban areas. History of multiple partners could be elicited from two cases in urban settings History of blood transfusion was given by 7 (4.51\%), 18 (11.61\%) of TB were tested for HIV. $77.35 \%$ from rural and $23.52 \%$ from urban areas (total 21.93\%) desired to know their HIV status.

Conclusion Maximum numbers of TB cases were in young agegroup, males, low socio-economic status particularly in urban slums with poor awareness level about HIV/AIDS. Coordinated efforts for implementation of the two programs for such population groups are required in controlling these diseases.

\section{P1-93 TRENDS IN THE BURDEN OF CARDIOVASCULAR DISEASES IN THE UK, 1961 TO 2011}

doi:10.1136/jech.2011.142976c.86

P Bhatnagar, ${ }^{*}$ P Scarborough, K Wickramasinghe. University of Oxford, Oxford, UK

Introduction Mortality from cardiovascular disease (CVD) has dramatically reduced over the past 50 years in the UK. While this trend should be celebrated, it is important to consider mortality alongside trends in morbidity to gain a full understanding of how healthcare resources and prevention schemes should be directed. 